\title{
The Ethnolinguistic Vitality of Naxi Language in Jinshan Bai Minority Township of Lijiang City
}

\author{
Fangfang Duan ${ }^{1, *}$
}

\author{
${ }^{1}$ Foreign Language Department, Lijiang Teachers College, Lijiang, Yunnan 650000, China \\ *Corresponding author. Email: duanfangfang5687@163.com
}

\begin{abstract}
As the important tool for human communication, language is a marker differing an ethnic group from another. The disappearance of any language will lead to the vanishing of cultural phenomena of human beings. The popularization of Chinese has impacted Naxi language. To assess the vitality of Naxi language can master the Naxi status and provide a reference for other ethnic minority languages in China. The paper firstly evaluated the vitality of Naxi language, explored the factors contributing to the status of Naxi, and finally proposed strategies for the maintaining of Naxi. The research employed the methodology of questionnaire, document analysis, interview, focus group discussion and observation. One hundred and thirty-three participants were involved in the survey and sixty-one were interviewed. Findings revealed the status of Naxi language in JT is at 'threatened' level. The sociolinguistic factors such as negative birth rate, the poor intergenerational language transmission, and the lack of materials for Naxi language education contributed to the threatened level of Naxi. The research finally proposed several countermeasures for the preservation of Naxi language. Keywords: Naxi language, vitality, contributing factors, preservation strategies
\end{abstract}

\section{INTRODUCTION}

At the commencement of 21 st century, with the acceleration of global economic development, the strengthening of the diffusion of universal languages, the functions of some vulnerable languages will gradually wane and eventually die out. According to Crystal (2007), one language is dribbling away in the world on average every two weeks [1]. Though Ethnologue reveals that there are above 7,097 languages in the world (Simmons \& Fennig, 2018) [9], researches have revealed that more than 3,405 residual languages are facing extinction at present (Grimes, 2000) [4]. Mufwene (1998) predicted that, unless decently protected, people will pay dearly price for the loss of languages spoken less by communities [5].

With the increasing awareness about language preservation, many researchers are focusing on the ethnolinguistic vitality of languages around the world. Coluzzi (2017) investigated the ethnolinguistic vitality of some of the most essential language varieties spoken in Malaysia, focusing on two languages as case studies [2]. Fortuno-Genuino (2011) conducted a fieldwork in the Philippines by employing domains of language use to evaluate Chabacano vitality [3]. Pareja (2014) developed a documentary lexicography on the list of Cavite City obsolescent Chabacano words to confirm the language status as to obsolete, definitely endangered, instable, nearly unstable, or currently being used [8]. In China, Sun (2006) conducted general research about the majority of ethnic languages in China, he applied UNESCO scales to finalize the ethnolinguistic vitality of ethnic languages, in which
Naxi language was positioned as the third scale "low vitality and threatened" [6]. Yu (2014) used three indicators of UNESCO scale. The result of the research showed that Naxi language in the Bai minority village of Jiuhe is at "with vitality" level [7].

Though many studies about ethnolinguistic vitality have been conducted abroad and at home, it is alarming to note that firstly most studies about Naxi language focused in Yulong Naxi Autonomous County and fewer studies have been done about the ethnolinguistic vitality of Naxi language in JT of Lijiang Ancient Town (LAT), which is a multilingual community and can to some extent predict the Naxi language vitality in Yunnan province. Secondly most studies focused on other endangered ethnic minority languages, few studies concentrated on Naxi ethnic minority language, which has the unique Dongba hieroglyphs. And thirdly most researchers in China conducted researches almost five years ago, which could be different from nowadays, the new research should be conducted immediately.

Generally, the study aimed to evaluate the vitality level of the Naxi language in the northwestern part of Yunnan province. Specifically, the study answered the following questions:

(1) What is the ethnolinguistic vitality level of Naxi language in JT of Lijiang City?

(2) What sociolinguistic factors are deemed contributory to the vitality level of Naxi language in JT?

(3) What strategies could be proposed to maintain Naxi language in LAT? 


\section{METHODOLOGY}

The study employed a qualitative semi-ethnographic research design as it evaluated the ethnolinguistic vitality of Naxi language in JT, excavated the sociological factors contributing to Naxi language status in JT, and proposed strategies for the preserving of Naxi language in JT. The data gathering in the study will employ questionnaire, interview, observation, field work, and focused group discussion (FGD).

The survey was conducted by administering a questionnaire in an interview style. The questionnaire had been translated from English to Chinese and checked by native Chinese speakers. It was not a self-administered questionnaire, but rather a face-to-face interview in Mandarin. Therefore, to improve the collection of questionnaire survey, the researcher gave guidance or support to them when they did not understand the questions instead of relying on the participants' self-interpretation. The study chose participants from four villages in JT of LATD. The participants taking part in UNESCO questionnaire were divided into four groups according to their age and were grouped into four according to age as follows: Group 1: 70 and above (great grandparent age); Group 2: 40-69 (grandparent age); Group 3: 20-39 (parent age); and Group 4: 5-19 (children age). The inclusion of 70 -year-old was based on the hypothesis that native speakers of Naxi are the elders who are 70 -year-old and above; while children at the age of five belong to the youngest generation where intergenerational language transmission may be observable (Genuino, 2011).

\section{RESULTS AND DISCUSSION}

\subsection{Research Problem One: Vitality Level of Naxi Language in Jinshan Bai Minority Township}

\subsubsection{Factor 1: Intergenerational Language Transmission (ILT)}

The researcher chose the Wenhong, Dewei, Qiyi and Xintuan village to conduct the household survey, altogether 133 native speakers were involved in the questionnaire. The Table 1 below shows the Number $(\mathrm{N})$ and Frequency (F) of Naxi language transmission in different generations based on the age groups. Table 1 presents the language transmission in different generations.
Table 1. Summary of Language Transmission of Native Naxi Based on Survey

\begin{tabular}{|c|c|c|c|c|c|c|c|}
\hline Age Group & Total & \multicolumn{2}{|c|}{ Grade 5 } & \multicolumn{2}{c|}{ Grade 4 } & \multicolumn{2}{c|}{ Grade 3 } \\
\hline & & $\mathrm{N}$ & $\mathrm{F}$ & $\mathrm{N}$ & $\mathrm{F}$ & $\mathrm{N}$ & $\mathrm{F}$ \\
\hline 70-older & 36 & & & 34 & $92 \%$ & 2 & $8 \%$ \\
\hline $40-69$ & 28 & & & 20 & $71 \%$ & 8 & $29 \%$ \\
\hline $20-39$ & 30 & & & 28 & $93 \%$ & 2 & $7 \%$ \\
\hline $5-19$ & 39 & & & 37 & $95 \%$ & 2 & $5 \%$ \\
\hline Total & 133 & & & 119 & $89 \%$ & 14 & $11 \%$ \\
\hline
\end{tabular}

The participants of the questionnaire suggest that Naxi is used in limited domains by some children, not all domains, which means that Naxi does not meet the condition of Grade 4. If one grade must be chosen for Naxi, it would be Grade 3 because the situation in JT matches one of the statements for Grade 3 that the main users of the language are the parental and older generations.

The conclusion can be proved by the FGD 1 with colleagues in Lijiang Teachers College (LTC) in which three teachers mentioned:

My nephew and niece cannot speak any other minority languages except Chinese.

Therefore, it can be concluded the transmission for Group A to Group C is good, but there are dangerous signals with the young generation. One participant in FGD 1 mentioned the reasons why young generations speak less Naxi:

Before going to kindergarten at 3 years old, my son used to speak Naxi language well, but his teacher said "speaking Naxi language will affect your learning of Chinese”. As time goes by, my son gradually spoke Naxi language less and less.

The FGD with teachers in LTC can be further testified by the interview with two participants in Qiyi village, they are at their 30s, they mentioned:

Our generation is ok, many children under 10 do not speak Naxi language even at home, most Naxi people in Dayan Town do not speak Naxi language any mores. (Interview 4)

Therefore, by combining the results, the researcher was more inclined to categorize the ILT of Naxi language into Grade 3-definitively endangered.

\subsubsection{Factor 2: Trends in Existing Language Domains}

Table 2. The Language Use Domains of the Participants

\begin{tabular}{|c|c|c|c|c|c|c|c|}
\hline $\begin{array}{c}\text { Age } \\
\text { Group }\end{array}$ & Total & \multicolumn{2}{|c|}{ Grade 4 } & \multicolumn{2}{c|}{ Grade 3 } & \multicolumn{2}{c|}{ Grade2 } \\
\hline & & $\mathrm{N}$ & $\mathrm{F}$ & $\mathrm{N}$ & $\mathrm{F}$ & $\mathrm{N}$ & $\mathrm{F}$ \\
\hline 70 -older & 36 & 36 & $100 \%$ & & & & \\
\hline $40-69$ & 28 & 13 & $46 \%$ & 15 & $54 \%$ & & \\
\hline $20-39$ & 30 & 16 & $53 \%$ & 14 & $47 \%$ & & \\
\hline $5-19$ & 39 & 21 & $54 \%$ & 16 & $41 \%$ & 2 & $5 \%$ \\
\hline Total & 133 & 86 & $64 \%$ & 45 & $34 \%$ & 2 & $2 \%$ \\
\hline
\end{tabular}


As shown in Table 2, the percentages of choosing Grade 4 are rarely used, but it is still used in the community public domain (religious ceremonies, community gatherings, etc.) and informal domains are 100, 46, 53, 54 respectively, with the average percentage 64 . The percentages of choosing Grade 3 are 54 in group B, 47 in group $\mathrm{C}$ and 41 in group $\mathrm{D}$, with the average percentage $34.2 \%$ of the 133PPs chose grade 2 'The language is used in limited social domains and for several functions.

In the FGD 2 with the participants, they mentioned the following:

The elders like my grandmother, most of them cannot speak Chinese, and the young generations like my son who usually communicate in Chinese, therefore, they two do not communicate with each other, because they two use different languages in family domains.

The analysis of the results shows that the people above 30 primarily use Naxi in informal domains, such as family domains, neighborhood domain, friendship domains, etc, while the young generations under 30 has almost shifted to Chinese. Therefore, the grade for Factor 2 would be Grade 4-multilingual parity.

\subsubsection{Factor 3: Response to New Domain and Media}

New domains and media are, for instance, TV, radio, broadcasting and the internet. For JT, the Grade can be categorized to 2 'The language is used in some new domains', such as TV programs, movies, TikTok, mobile phones etc. When the survey was conducted in villages, participants mentioned that Naxi programs has been involved in some new domains, just as the Table 3 shown below. Table 3 shows the new domains and media used in JT.

Table 3. New Domains and Media Used in JT

\begin{tabular}{|c|c|c|c|c|c|}
\hline $\begin{array}{c}\text { Age } \\
\text { Group }\end{array}$ & Total & Grade 4 & Grade 3 & Grade 2 & Grade1 \\
\hline & & $\mathrm{N}$ & $\mathrm{N}$ & $\mathrm{N}$ & $\mathrm{N}$ \\
\hline $\begin{array}{c}70- \\
\text { older }\end{array}$ & 36 & 3 & 15 & 15 & 3 \\
\hline $40-69$ & 28 & 1 & 9 & 15 & 3 \\
\hline $20-39$ & 30 & & 12 & 16 & 2 \\
\hline $5-19$ & 39 & & 12 & 22 & 5 \\
\hline Total & 133 & 4 & 48 & 68 & 13 \\
\hline
\end{tabular}

Based on the researcher's interview and group discussion with participants, Grade 2 is better appropriate for the situation in JT. During the first interview with a tour guide in Wenhong village, she said:

We can often watch Naxi TV programs on Yulong TV and Ancient Town TV, such as the Speaking Naxi Camp, Yulong news broadcast which circulates the news the whole day, Tik Tok, Kuaishou (short-video app) and so on.

The universality of Naxi language is used in new domains can be further verified by the focus group discussion in Qiyi village as shown by the excerpt that follows:

There are some Naxi locals creating their own sitcoms in their quadrangle courtyard.

Though the survey results and focus group discussion proved that Naxi language is widely used in new domains, compared with the total population of the Naxi people in JT, the Grade for Response to New Domains and Media of Naxi language should be rated to 2Coping 'The language is used in some new domains.'

\subsection{Research Problem Two: Sociolinguistic Factors Contributory to the threatened Level of Naxi Language}

\subsubsection{Birth Rate}

Though the absolute number of people speaking Naxi is more than 100,000 , it is worth noting that the birth rate of Naxi people is growing negatively. The population development of an ethnic minority is influenced by the national population policy and the background of economic and social development, in the past 60 years, the population development of Lijiang area is basically consistent with the country's population development. According to the third national census bulletins, the Naxi population has shown negative growth. If the growth rate slows down, its proportion in the total population of the country and the proportion of neighboring ethnic groups will continue to decline.

In the first interview with the tour guide in Wenhong, she was also very concerned about the impact of negative population growth on Naxi language:

The "Chinese fever" has been a thing of recent years. In contrast, the Naxi population is growing negatively, thus I think we should encourage the Naxi to increase their birth rate.

To sum up, the negative birth rate of birth rate has constituted one essential factor contributory to the threatened level of Naxi language.

\subsubsection{Poor Intergenerational Language Transmission}

In JT, at least people above 30 can frequently use Naxi, and their best-spoken language is Naxi rather than Chinese. However, the Naxi language is threatened because the young generation has taken Chinese as the best-spoken and the most frequently used language. 
Based on the interview and groups discussion with participants, they mentioned that their grandchildren were good in listening but their speaking ability is not as good as their parents' generation:

Children speak less Naxi language after going to kindergarten, they indeed speak less Minority language. Because they have been taught Chinese since they went to kindergarten, they go to the kindergarten will speak Chinese frequently. (Interview 3)

It can be seen that the Naxi language is inclined to be threatened and the main reason can be due to the young generations speaking less and less and some do not even speak Naxi with their parents at home as mentioned in FGD1:

No matter what language I talk with him, he will always reply me in Naxi.

\subsubsection{Lack of Materials for Language Education and Literacy}

Though Dongba symbols are the written language of Naxi minority, the language use range is only limited to Dongba (Naxi priest). Dongba characters have not been popularized in people's daily lives and the Naxi language is more considered as a word-of-mouth language although some Chinese government language experts, scholars, and foreigners have created different systems of literacy, which is considered complicated and inaccurate. Some colleagues in Lijiang Teachers College tried to pronounce the words annotated by International Phonetic Alphabet but failed to do so. Furthermore, the created annotated literacy systems are not widely used in communities and can only be found in Research Center, Bookstore or few members of the community. The data collected show the literacy education in the Naxi language is not any part of the school curriculum in JT. Therefore, the lack of Naxi orthography in communities has been a great threat to the vitality of Naxi language.

\subsection{Research Problem Three: Strategies proposed to preserve Naxi language}

Several potential strategies for the language maintenance of Naxi in JT are proposed.

\subsubsection{Giving Extra Grades in Students' College Entrance Examination (CEE)}

As the country's largest exam, some people say that CEE is not only an exam determining the allocation of social resources, but also the only springboard for poor children in their lives to change their fate. Only through the CEE can the poor students break away from their current environment, and CEE is the opportunity especially for poor students. Thus, it can be seen the importance of CEE for the broad masses of test-takers.

Participants also mentioned the practical effect in giving points, they stated:

There is a policy that should be implemented immediately. Only students realize the practical benefits can motivate them to learn Naxi language. There must be some mandatory measures to create a language learning environment for him. (FGD 1)

Although this strategy is more utilitarian, it can quickly attract more young people to join the ranks of national cultural heritage and protection, and then gradually change the goal from getting grades in CEE to the heartfelt love for national language, to ask them to learn Naxi language, to use Naxi language in daily life, to inherit and spread Naxi language as their lifelong goal, and to contribute their light and enthusiasm to the considerable vitality of the national language.

\subsubsection{Increasing the Practical Bilingual Education in Schools of JT}

It is necessary to learn the advanced culture of other nationalities mainly Han nationality and master the mainstream language as a communication tool. However, if the impact of modern civilization exceeds the carrying limit of Naxi language and culture, these intangible cultural heritages may disappear irreversibly. We should not ignore the legacy of the Naxi language as mother tongue and Naxi culture because of the economic prosperity brought by tourism. Mother tongue learning must start with children, even from kindergarten. The participants in FGD 1 mentioned:

Naxi language must be taught in primary and secondary schools, should be especially and vigorously promoted in kindergartens, let them immerse in such a big environment to urge them to speak Naxi.

In terms of higher education, the support for Naxi research needs to be expanded. Colleges and universities in Lijiang can also set up Naxi culture and Naxi language as minor courses, so that students of the ethnic groups and interested students from all over China have the chance to learn and understand Naxi culture and Naxi language.

\subsubsection{Formulating Positive Language Propaganda Policies and Regulations}

The Yunnan Provincial Government has issued the Regulations on the Protection of National and Folk Traditional Culture of Yunnan Province and the Regulations on the Protection of Dongba Culture of Naxi Autonomous County in Lijiang, Yunnan Province, which indicates that the protection of Dongba culture of 
Naxi has embarked on the road of legalization. The external environment of language plays a key role in the use and development of language. This external environment can include language policy measures and economic environment. The maintenance of language also needs the protection of law. The relevant government departments of Lijiang can make regular investigations on the use of Naxi language and cultural heritage, based on investigation and community participation, to formulate of laws and regulations to ensure the use of the Naxi language and to consciously improve its language status, and to call for the Naxi people and people living in Lijiang to use it in their daily life.

\section{CONCLUSION}

The researcher employed three indicators of UNESCO scale to evaluate Naxi language status by targeting at four villages in JT of Lijiang Ancient Town, the findings revealed Naxi language was in a threatened level, and the factors resulting in the present levels of Naxi language are poor intergenerational language transmission, negative birth rate, and lack of materials for language education and literacy. And the researcher proposed countermeasures for the maintenance of Naxi language. The present study provides theoretical framework reference to researchers of ethnic minorities, the result of the study proves to be objective and credible. The results of the study also showed Naxi language in a threatened level which could raise authorities' attention for the maintenance of Naxi language. Furthermore, the combination of multiple research methods could reflect Naxi language status in different ways, which is conductive to the comprehensiveness and objectiveness of the studied languages. The researcher believes that if the countermeasures could be implemented, the future of Naxi languages will become brighter and brighter.

\section{REFERENCES}

[1] D. Crystal, "By hook or by crook: A journey in search of English,” The Overlook Press, 2007.

[2] P. Coluzzi, "The vitality of minority languages in Malaysia," Oceanic Linguistics, 2017, 56(1), pp.210225.

[3] C. Fortuno-Genuino, "Is Chabacano dying?", Selected papers from Philippine-Spanish Friendship Day conferences, 2009, pp. 1-25. National Historical Commission of the Philippines.

[4] B. F. Grimes, "Ethnologue (14th ed.)," SIL International, 2000.

[5] S. Mufwene, "The ecology of language: New imperatives in linguistic curricula," Studies in the Linguistic Sciences, 1998, 28(2).

[6] H. K. Sun, "A study on the ranking of Chinese minority language vitality," Journal of Guangxi University for Nationalities, 2006, (05), pp. 6-10.

[7] J. Z. Yu, "Study on the Naxi native language vitality of Bai nationality in Jiuhe Bai nationality township," Journal of Qiannan Normal University for Nationalities, 2014, 34(05), pp. 9-13.

[8] T.Pareja, "The Chabacano of Cavite City," 2014, Unpublished research.

[9] F.G.Simons and D.C.Fennig, "Ethnologue: Languages of the world," 21st ed. Dallas, Texas: SIL International, 2018. 